\title{
The downstream of tyrosine kinase 7 is reduced in lung cancer and is associated with poor survival of patients with lung cancer
}

\author{
GANG CHEN ${ }^{1-4 *}$, HEFEN YU $^{1-3 *}$, LUCY SATHERLEY $^{4}$, CATHERINE ZABKIEWICZ $^{4}$, JEYNA RESAUL $^{4}$, \\ HUISHAN ZHAO ${ }^{1-4,6}$, HU MU ${ }^{5}$, XIUYI ZHI ${ }^{5}$, JUNQI HE ${ }^{1-3}$, LIN YE ${ }^{4}$ and WEN G. JIANG ${ }^{2-4}$
}

\begin{abstract}
${ }^{1}$ Department of Biochemistry and Molecular Biology, School of Basic Medical Sciences, Capital Medical University;
${ }^{2}$ Cancer Institute of Capital Medical University; ${ }^{3}$ Beijing Key Laboratory for Cancer Invasion and Metastasis Research,

Beijing 100069, P.R. China; ${ }^{4}$ Cardiff-China Medical Research Collaborative, Cardiff University School of Medicine,

Heath Park, Cardiff, CF14 4XN, UK; ${ }^{5}$ Xuanwu Hospital Capital Medical University, Beijing 100053; ${ }^{6}$ Central Laboratory, Yantai Yuhuangding Hospital, Affiliated Hospital of Medical College Qingdao University, Yantai, Shandong 264000, P.R. China
\end{abstract}

Received December 9, 2016; Accepted March 3, 2017

DOI: $10.3892 /$ or.2017.5538

\begin{abstract}
The downstream of tyrosine kinase 7 (DOK7) is an adaptor protein mediating signalling transduction between receptors and intracellular downstream molecules. Reduced expression of DOK7 has been observed in breast cancer. The present study aimed to investigate the role played by DOK7 in lung cancer. The expression of DOK7 at both mRNA and protein levels was evaluated in human lung cancer. A reduced expression of DOK7 transcripts was seen in lung cancers compared with normal lung tissues. Kaplan-Meier analyses showed that the reduced expression of DOK7 was associated with poorer overall survival and progression-free survival of patients with lung cancer. A further western blot analysis revealed a predominant expression of DOK7 isoform 1 (DOK7V1) in normal lung tissues, which was reduced in lung cancer. Forced overexpression of DOK7V1 in lung cancer cell lines, A549 and H3122 resulted in a decrease of in vitro cell proliferation and migration, while adhesion to extracellular matrix was enhanced following the expression. In conclusion, DOK7 was reduced in lung cancer and reduced DOK7 expression was associated with poorer survival. DOK7 isoform 1 plays an inhibitory role on the proliferation and migration of lung cancer cells in which Akt pathway may be involved.
\end{abstract}

\section{Introduction}

Lung cancer is the leading cause of cancer death worldwide, accounting for $19 \%$ of total cancer deaths. The ratio of

Correspondence to: Professor Wen G. Jiang, Cardiff China Medical Research Collaborative, Cardiff University School of Medicine, Academia Avenue, Cardiff, CF14 4XN, UK

E-mail: jiangw@cardiff.ac.uk

*Contributed equally

Key words: downstream of tyrosine kinase 7, lung cancer, survival, adhesion and migration mortality to incidence is 0.87 , reflecting the highly fatal nature of the disease, despite advances in surgery, chemotherapy and radiotherapy $(1,2)$. Lung cancer is categorised into two major groups: small cell lung cancer (SCLC) and non-small cell lung cancer (NSCLC). NSCLC comprises the majority of lung cancers, and is more often diagnosed at an advanced stage with poor survival (3). More intensive and effective studies are required, to identify new diagnostic, prognostic and therapeutic markers, such that improvements can be made in early diagnosis and targeted treatments.

From primary tumour to metastasis, cancer cells rely on diverse signalling pathways to promote their proliferation, survival, invasion and subsequent dissemination through lymphatic and blood vessels. Receptor tyrosine kinases (RTKs) contain an extracellular domain for ligand binding, a transmembrane helix and a cytoplasmic tyrosine kinase domain, and an intracellular juxtamembrane regulatory region (4). RTKs play a pivotal role in regulation of cellular processes including cell survival, cell proliferation, cell differentiation, cell migration and cell cycle control $(5,6)$. Dysregulation of RTKs, including autocrine activation, chromosomal translocations, overexpression of RTKs or gain-of-function mutations, occurs in cancer (4).

The downstream of kinase (DOK) protein family, comprising seven members, mediates intracellular signal transduction downstream of RTKs (7-9). DOK proteins share a structural topology characterised by an NH2-terminal pleckstrin homology $(\mathrm{PH})$ domain, a central phosphotyrosine binding (PTB) domain, followed by SH2 target motifs in the carboxyl-terminal $(7,10)$. The DOK proteins are divided into three subgroups; DOK 1-3 are primarily expressed in haematopoietic tissues (11), DOK 4-6 are predominantly expressed within the nervous system $(12,13)$, and DOK7 is mainly expressed in skeletal muscle and the heart, and plays a distinct role in comparison with the other members (14).

Deregulation of DOK proteins have been observed in various malignancies. DOK1, DOK2, and DOK3 have been shown to cooperatively suppress aggressive histiocytic sarcoma in a mouse model (11). DOK2 has been suggested as a predictive factor for poor prognosis of gastric cancer $(15,16)$. 
In lung cancer, loss of DOK1, DOK2 and DOK3 resulted in aberrant proliferation of alveolar type II cells and bronchoalveolar stem cells, with subsequent development of lung cancer in a mouse model (7). Reduced expression of DOK2 was also seen in human lung cancers, and a forced overexpression of DOK2 inhibited the proliferation of lung cancer cells (7).

DOK 7 is a cytoplasmic adaptor protein that is necessary for muscle specific kinase (MuSK) activation and Acetylcholine receptor (AChR) clustering, which are indispensible for neuromuscular synapse function $(17,18)$. DOK7 interacts with the juxtamembrane region of MuSK via its PTB domain, although both PH and PTB domains are required for MuSK activation (18-20). DOK7 is not only a substrate of MuSK, but can also activate MuSK kinase activity (21). Agrin requires DOK7 to activate MuSK and DOK7 is also necessary for the correct localisation of MuSK in muscle (22). Overexpression of DOK7 was associated with increased activation of MuSK and neuromuscular junction formation (22). On the other hand, DOK7 expression enhanced MuSK activation and restored neuromuscular junction formation in agrin-deficient mice $(23,24)$. In addition to its profound function at neuromuscular junction, a recent study has revealed a hypermethylation of DOK7 promoter in breast cancer (25). However, the expression and biological function of DOK7 in other malignancies, particularly lung cancer, remains largely unknown.

In the present study, we aimed to evaluate DOK7 expression in lung cancer and the involvement in disease progression. Our current study revealed predominant expression of DOK7 protein isoform 1 (DOK7V1) in normal lung tissues, which was reduced in lung cancers. We further investigated its regulatory role of cellular functions in lung cancer cell lines using an overexpression plasmid vector carrying a DOK7V1 coding sequence.

\section{Materials and methods}

Cell culture. Lung cancer cell lines, A549 and H3122 were purchased from the American Type Culture Collection (ATCC, Manassas, VA,USA).Cells were routinely cultured in Dulbecco's modified Eagle's medium (DMEM) with L-glutamine (Thermo Fisher Scientific, Hudson, NH, USA) supplemented with $10 \%$ fetal bovine serum (ExCellBio, Shanghai, China), in an incubator at $37^{\circ} \mathrm{C}, 5 \% \mathrm{CO}_{2}$ and $95 \%$ humidity.

Construction of DOK7 expression vectors and transfection: the DOK7-Flag and pEnter (vector) plasmids were purchased from Vigene Biosciences (Rockville, MD, USA). Purified DOK7V1 transgenes and control plasmid vectors were transfected into A549 and H3122 cells, respectively, using Neofect ${ }^{\mathrm{TM}}$ DNA transfection reagent (Neofectbiotech Co., Ltd., Beijing, China).

Western blot analysis. Protein concentrations were determined with the BCA Protein Assay kit and a spectrophotometer (both by Thermo Fisher Scientific). Equal amounts of protein sample were separated using $10 \%$ sodium dodecyl sulfatepolyacrylamide gel electrophoresis (SDS-PAGE) and blotted onto a nitrocellulose membrane. The membranes were blocked with 5\% skimmed milk in TBS buffer for $1 \mathrm{~h}$. Proteins were then probed with anti-FLAG monoclonal antibody (Sigma, St.Louis, MO,USA), anti-DOK7 antibody (Abcam, Cambridge,
MA, USA), monoclonal mouse anti-human GAPDH antibody (Santa Cruz Biotechnology, Inc., Dallas, TX, USA), anti-Akt antibody (Sigma) and anti-phosphorylated-Akt (Ser473, Cell Signaling Technology, Danvers, MA, USA) and corresponding peroxidase-conjugated secondary antibody. Protein bands were visualised and analysed using Vilber Fusion Fx5 (Vilber, Marne La Vallée, France).

In vitro cell function assays. Cells were detached from the culture dish and cell density (per millilitre) was counted. Cells were then seeded onto a 96-well plate at a density of 3000 cells in $200 \mu \mathrm{l}$ of culture medium. Five plates were set up to obtain a cell density reading, following incubation up to 4 days. After the incubation, culture medium was removed and replaced with $100 \mu 1$ of $10 \%$ CCK-8 (Cell Counting Kit-8, Dojindo, Japan). Absorbance at $450 \mathrm{~nm}$ was then determined after an incubation at $37^{\circ} \mathrm{C}, 5 \% \mathrm{CO}_{2}$ for $1 \mathrm{~h}$ using a spectrophotometer (Thermo Fisher Scientific).

In vitro tumour cell Matrigel adhesion assay. Matrigel (5 $\mu \mathrm{g})$ was added to $100 \mu \mathrm{l}$ of serum-free medium to coat the culture surface of each well of a 96-well plate and dried in an oven to form an artificial basement membrane. This membrane was then rehydrated in $100 \mu \mathrm{l}$ of sterile distilled water for $40 \mathrm{~min}$ before seeding 20,000 cells/200 $\mu \mathrm{l}$ culture medium into each well. After an incubation of $40 \mathrm{~min}$, the medium was removed and non-adherent cells were washed off with $150 \mu 1$ of PBS buffer. Adherent cells were then fixed in $4 \%$ formaldehyde $(\mathrm{v} / \mathrm{v})$ in PBS for 30 min before being stained in $0.5 \%$ crystal violet solution ( $\mathrm{w} / \mathrm{v})$ in distilled water. Crystal violet staining was dissolved with $10 \%$ acetic acid and the absorbance at $570 \mathrm{~nm}$ was then determined using the spectrophotometer.

In vitro tumour cell migration assay. Cells $\left(1 \times 10^{6} /\right.$ well) were seeded to a 6-well plate and cultured in the incubator overnight, then scratched with a $200 \mu \mathrm{l}$ pipette tip to create a wound and washed twice with PBS to remove floating cells. The cells were photographed every $6 \mathrm{~h}$ using an inverted microscope over a period up to $24 \mathrm{~h}$. The size of the wounds were subsequently measured with ImageJ software.

\section{Results}

Expression of DOK7 in lung cancer. We initially evaluated the expression of DOK7 in lung cancer by analysing its levels in a cohort of lung cancer tissue deposited at The Cancer Genome Atlas (TCGA) adenocarcinoma database. Reduced transcript levels of DOK7 were seen in the lung cancer compared with normal lung tissues (Fig. 1A). We also analysed DOK7 expression levels in another cohort of lung cancer which had paired adjacent normal lung tissues (GSE19804, 240633_at) (26). The reduced expression of DOK7 was also seen in these lung cancer samples in comparison with the paired adjacent normal lung tissues, $\mathrm{p}<0.001$ (Fig. 1B).

Reduced expression of DOK7 was associated with the survival of patients with lung cancer. To further clarify the role played by this molecule in lung cancer, we analysed the association between DOK7 expression (Affy ID 240633_at) and survival of patients with lung cancer using an online 

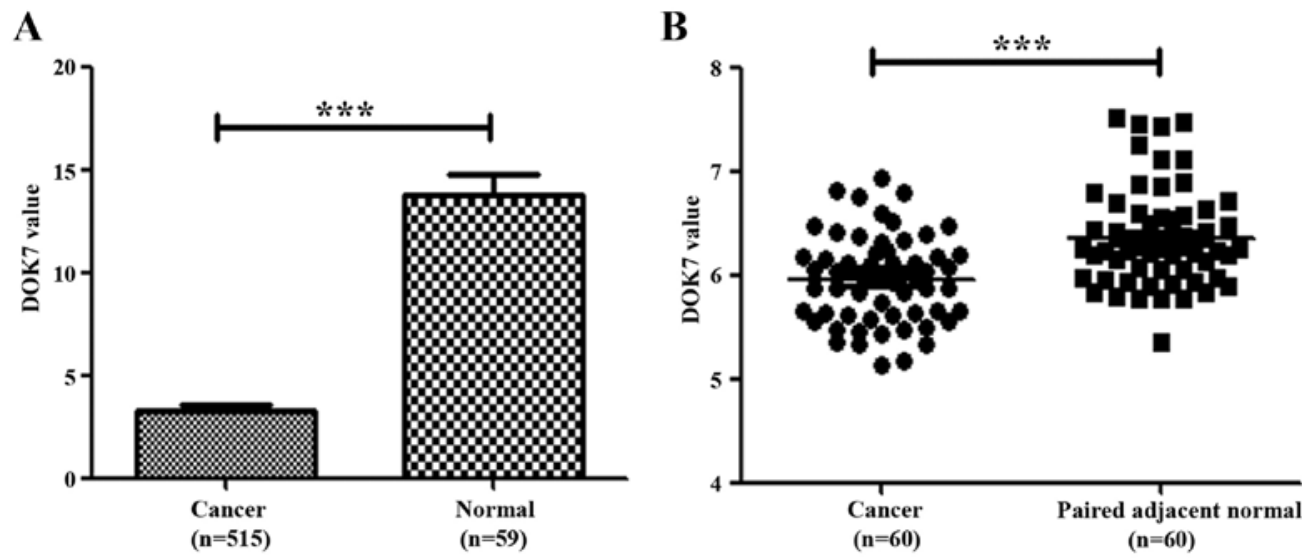

Figure 1. Expression of DOK7 transcripts in human lung cancer. (A) Transcript levels of DOK7 were analysed in a cohort of lung cancers (515 cases) compared with normal lung tissues (59 cases) which were obtained from a collection of gene expression array data at TCGA (http://www.cbioportal.org/data_sets.jsp). (B) DOK7 expression in lung cancer compared with paired adjacent normal lung tissues (GSE19804) was analysed using paired t-test. ${ }^{* * *}$ p $<0.001$.
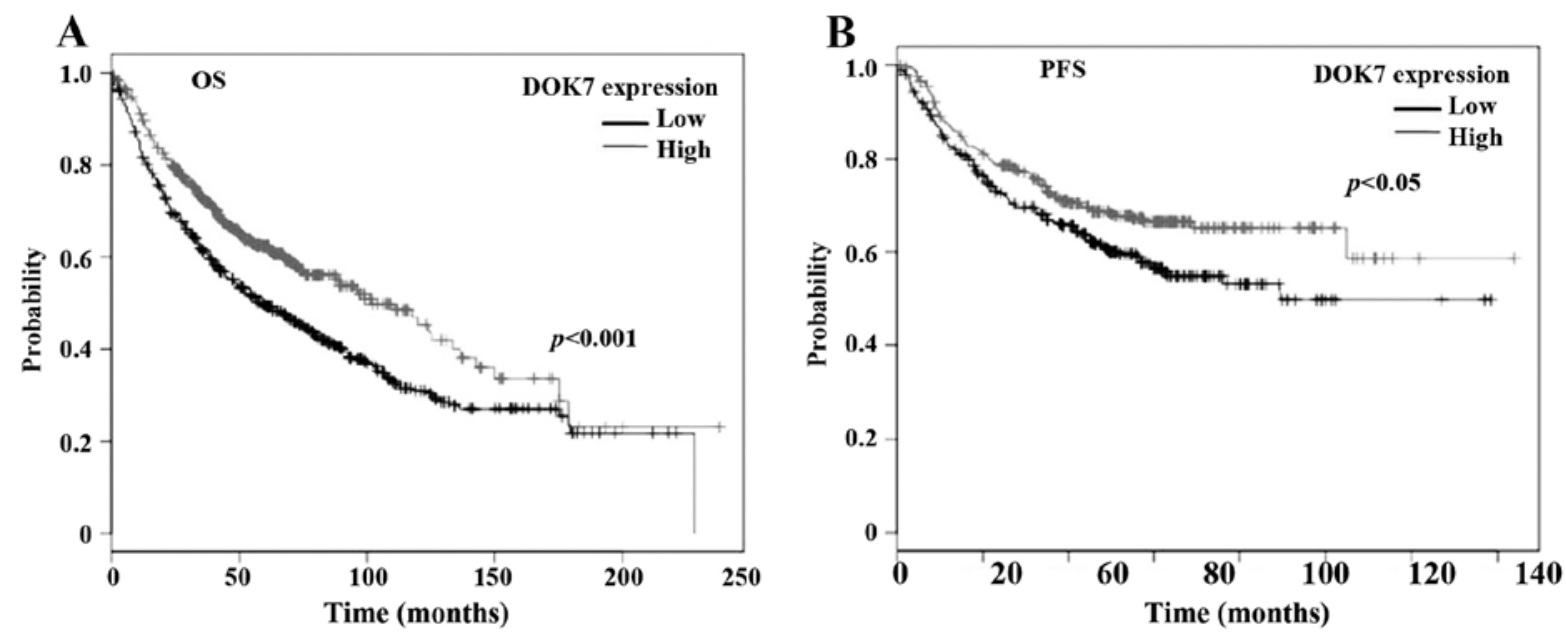

Figure 2. DOK7 expression and survival of patients with lung cancer. Association between DOK7 expression and overall survival (OS, A) and progression-free survival (PFS, B) was analysed using an online Kaplan-Meier survival analysis (KMplot, http://kmplot.com/analysis/). An auto-selected cut-off value of 115 was employed in the analysis.

Kaplan-Meier survival analysis tool (KMplot, http://kmplot. com/analysis/) (27). The Kaplan-Meier analyses showed that the reduced expression of DOK7 was significantly associated with poorer overall survival (OS), $\mathrm{p}<0.001$ (Fig. 2A), in a cohort of 1145 cases of lung cancer. The reduced DOK7 expression was also associated with poor progression-free survival (PFS) of a cohort of 596 patients with lung cancer, $p=0.019$ (Fig. 2B).

Expression of DOK7 protein isoforms in human lung cancer tissues. The expression of DOK7 protein isoforms was examined in a cohort of lung cancer tissues using western blot analysis. DOK7 isoform 1 (DOK7V1) was predominantly expressed in normal lung tissues in comparison to the expression of its isoform 2 (DOK7V2) (Fig. 3). DOKV1 exhibited low expression in lung cancer tissues compared with the paired adjacent normal lung tissues $(\mathrm{p}<0.01, \mathrm{n}=12)$. DOK7V2 protein was either weakly expressed or absent from both lung cancer tissues and adjacent normal lung tissues (Fig. 3).

Overexpression of DOK7V1 exhibited an inhibitory effect on proliferation of lung cancer cell lines. To further study the role of DOK7 in lung cancer, we constructed an overexpression plasmid vector which carried the coding sequence of DOK7V1. Overexpression of DOK7V1 was performed in A549 and H3122 lung cancer cell lines. Following transfection and selection, transfected cell lines were verified for the expression of DOK7V1 using western blotting (Fig. 4A). The overexpression of DOK7V1 resulted in a reduction of in vitro cell proliferation of A549 cells compared with the control cells which were transfected with the empty plasmid vector (Fig. 4B). In comparison with the A549 cells, H3122 exhibited less response to the inhibitory effect on proliferation by DOK7V1 overexpression (Fig. 4C).

Influence of DOK7V1 on adhesion and migration of lung cancer cells. The overexpression of DOK7V1 resulted in an increase in cell adhesion to an artificial matrix gel in both A549 and H3122 lung cancer cell lines (Fig. 5). In contrast to the promotion of cellular adhesion, a reduction was seen in the migration of both A549 and H3122 cell lines following the overexpression of DOK7V1 in comparison with the respective control cells (Fig. 6). 

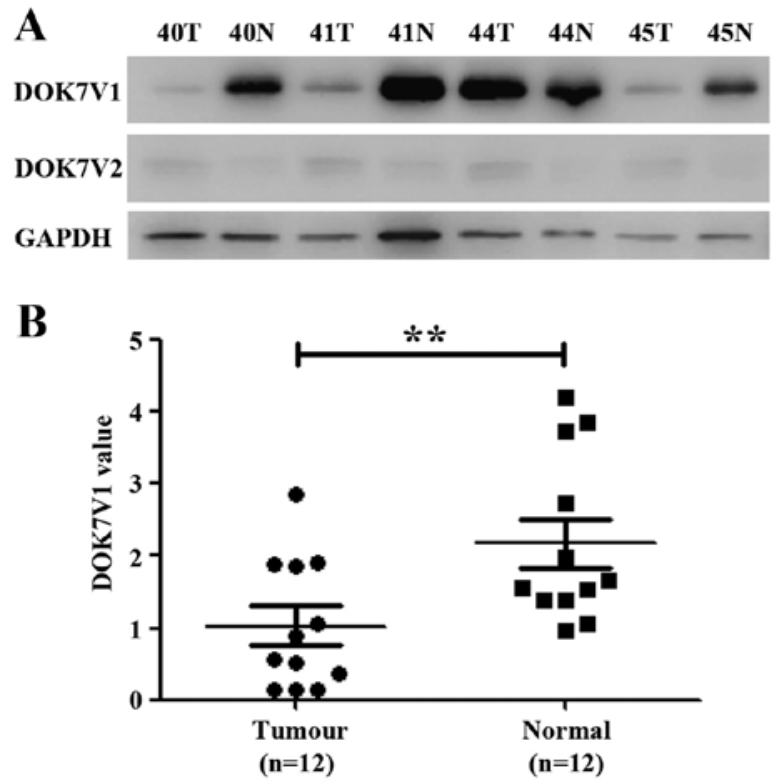

C

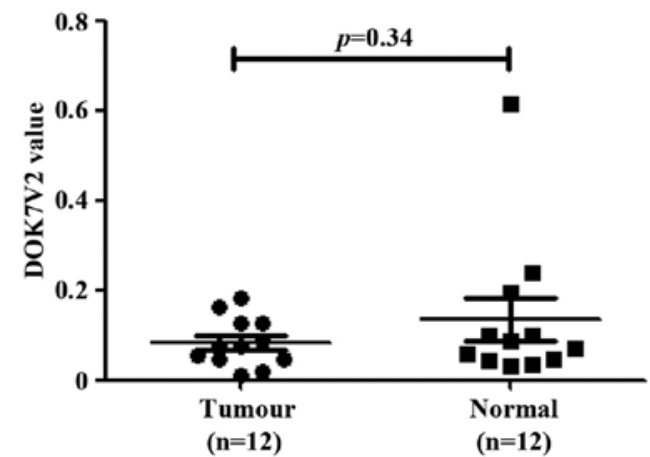

Figure 3. DOK7 protein isoform-1 (DOK7V1) and -2 (DOK7V2) were determined in 12 lung cancer tumours together with paired adjacent normal lung tissues using western blotting. (A) Shown are representatives from the 12-paired lung cancer samples of western blotting. A semi-quantitative analysis was performed using ImageJ. Shown are values of band integrated density normalised against the corresponding loading control (GAPDH), DOK7V1 (B), and DOK7v2 (C), respectively. ${ }^{* *} \mathrm{p}<0.01$.

Phosphorylation of Akt was altered in the DOK7V1 overexpression lung cancer cell lines. Following the functional assays, we performed some experiments to examine involvement of Akt and ERK pathways which have shown associated with functions of certain DOK proteins (7). A decrease of Akt phosphorylation was seen in both A549 and H3122 cell lines which overexpressed the DOK7 variant 1 while the total Akt protein levels remained similar in comparison with their corresponding controls (Fig. 7).

\section{Discussion}

This is the first study regarding the downstream of tyrosine kinase 7 (DOK7) in lung cancer, in which we determined protein isoform -1 and -2 expression levels in human lung cancer tissues, compared with paired adjacent normal lung tissues. In the present study, a reduced transcript level of DOK7 was seen in lung cancer in comparison to normal lung tissues or paired adjacent normal lung tissues. Western blot analyses further revealed that one of the DOK7 protein
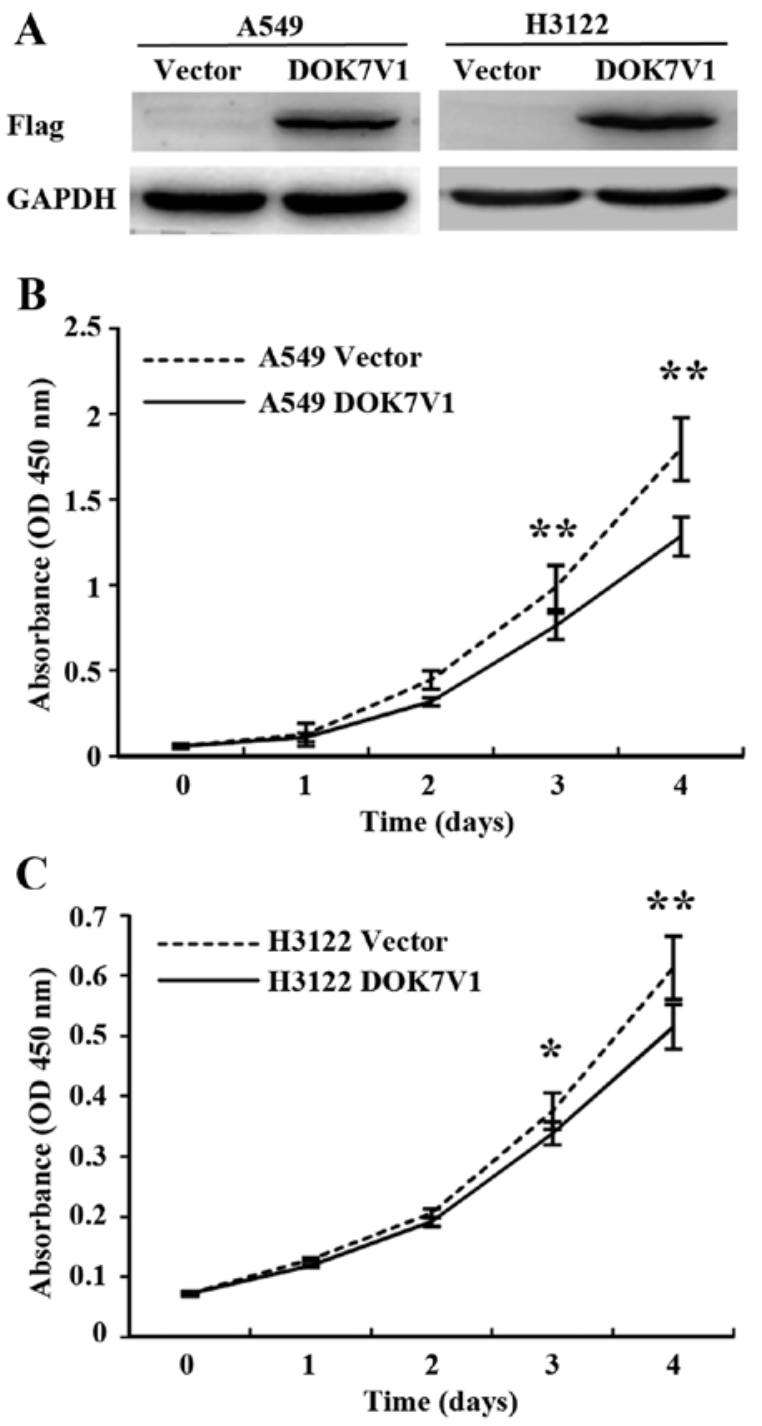

Figure 4. Overexpression of DOK7V1 in lung cancer cell lines and the effect on in vitro proliferation. (A) The forced overexpression of DOK7V1 was confirmed using western blotting. The influence of DOK7V1 was determined using an in vitro colorimetric growth assay for A549 (B) and H3122 (C) lung cancer cell lines in comparison with their corresponding control cell lines which were transfected with the empty plasmid vector. Six repeats were performed for each cell line in each experiments. Three independent experiments were carried out. Shown are representative data. Error bars represent standard deviations. ${ }^{*} \mathrm{p}<0.05 ;{ }^{* *} \mathrm{p}<0.01$.

isoforms, isoform 1 (DOKV1) was predominantly expressed in the normal lung tissues, and was reduced in lung cancers.

Recent studies of other DOK proteins in lung cancers have demonstrated that DOK1, DOK2 and DOK3 are reduced or absent in lung cancer $(7,28)$. In mouse models, loss of DOK1, DOK2 and DOK3 resulted in an increased proliferation of alveolar type II cells and bronchoalveolar stem cells leading to a development of lung cancer particularly in a triple knockout mouse model (7). A reduced expression of DOK2 was also observed in human lung adenocarcinomas, whilst DOK2 overexpression resulted in an inhibition of proliferation of lung cancer cells (7). Chromosome 8p21.3 where DOK2 gene is located, is one of the common regions deleted in human lung cancer (28). The loss of one copy of the DOK2 gene was seen in $37 \%$ of human lung adenocarcinoma and $33 \%$ of 

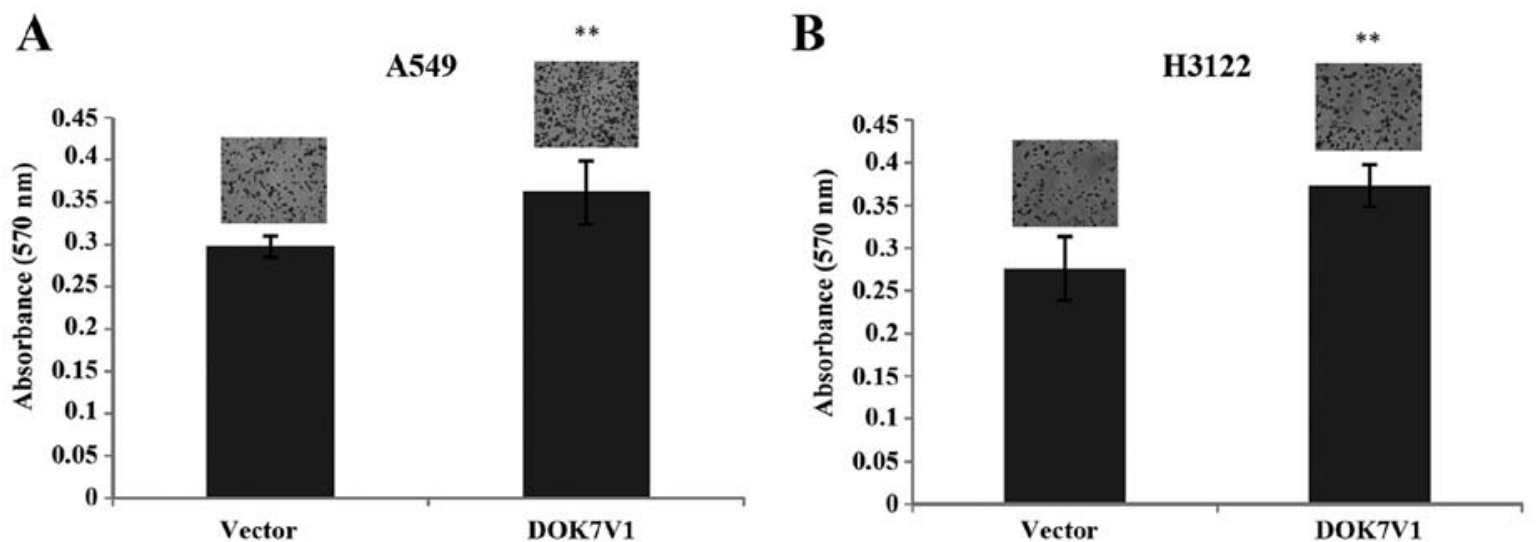

Figure 5. DOK7V1 overexpression and adhesiveness of lung cancer cell lines. The adhesion to extracellular matrix was determined using an adhesion assay for A549 (A) and H3122 (B) cell lines, respectively. Six repeats were included for each cell lines in every adhesion assay and three independent experiments were performed. Shown are representative data. ${ }^{* *} \mathrm{p}<0.01$.

A

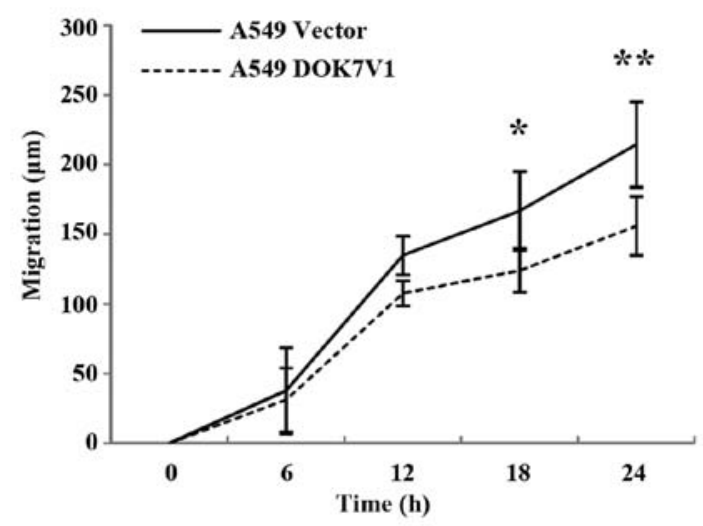

C

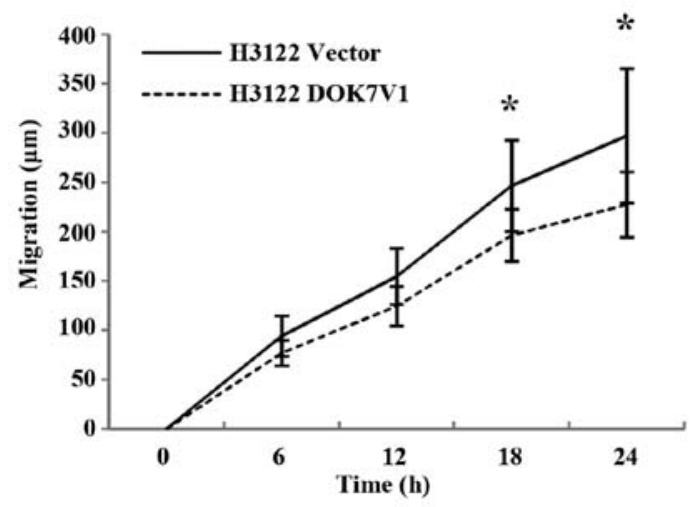

B

$\mathbf{0 h}$
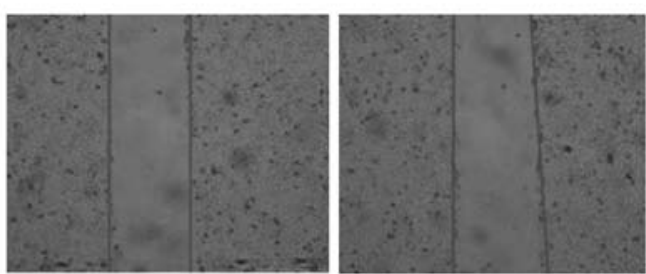

$24 \mathrm{~h}$

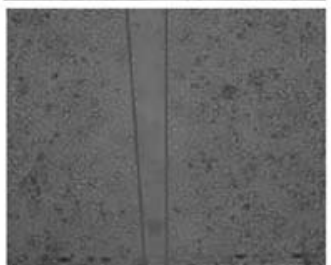

Vector

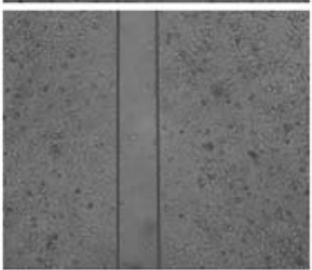

DOK7V1

D

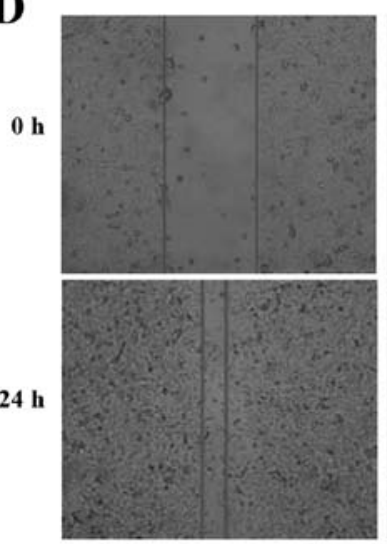

Vector
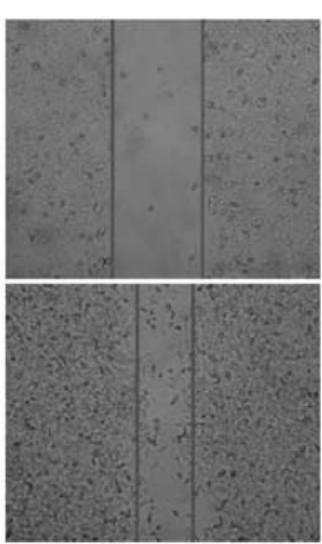

DOK7V1

Figure 6. Migration of DOK7V1 overexpression cell lines were determined using a wound assay for A549 (A and B) and H3122 (C and D). Three independent experiments were performed. Error bars are standard deviations. ${ }^{*} \mathrm{p}<0.05$ and ${ }^{* *} \mathrm{p}<0.01$.

human non-small cell lung cancers (7). Reduced expression of DOK2 was seen in both primary lung adenocarcinomas and lymph node metastases (7). In contrast to the observation in mouse models, no differential expression of DOK1 and DOK3 was seen in human lung cancer in comparison with normal lung tissues, although a reduced DOK3 was noted in lymph node metastases compared with its expression in the primary tumours (7).

A recent study of DOK7 in breast cancer has demonstrated that $\mathrm{CpG}$ islands of DOK7 promoter are hypermethylated leading to a downregulation its expression (25). These studies indicate that an inhibitory role may be played by DOK 
A549

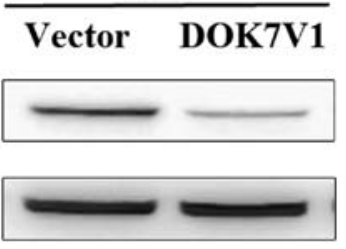

\section{GAPDH}

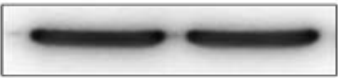

Figure 7. Phosphorylated Akt in DOK7 variant 1 overexpressing lung cancer cell lines. A reduced level of phosphorylated Akt was seen in both lung cancer cell lines with overexpression of the DOK7 variant 1 compared with the corresponding control using western blot analysis. GAPDH and Akt were used as loading controls.

proteins in cancer. Downregulation or loss of DOK proteins may occur in cancer cells due to gene deletion or hypermethylation. Additionally, mutations in DOK7 are a common cause of congenital myasthenic syndrome (29). However, whether the reduced expression or loss of DOK7 in lung cancer results from these events is yet to be investigated. The role of DOK7 mutation in cancer also requires investigation.

Kaplan-Meier survival analyses of DOK7 transcripts in lung cancer show that the reduced transcript levels of DOK7 were associated with both poorer OS and PFS. It suggests that DOK7 plays an inhibitory role during disease progression and relapse of lung cancer. To further explore this hypothesis, we constructed a plasmid vector carrying a DOK7V1 coding sequence. Overexpression of this isoform resulted in a decreased proliferation of both A549 and H3122 lung cancer cell lines. In keeping with the inhibitory role of DOK proteins in lung cancer, forced expression of DOK2 in a lung cancer cell line has been seen to reduce growth rate and both Akt and Erk phosphorylation (7).

It has been suggested that certain DOK proteins play an inhibitory role in signal transduction of some RTKs, therefore the loss of these DOK proteins leads to a promotion of the RTK-mediated proliferation. Further study will elucidate the role played by DOK7 in RTK signalling and the disease progression of lung cancer.

In addition to its effect on cell proliferation, a similar inhibitory effect was also observed in migration of both A549 and $\mathrm{H} 3122$ cell lines following the overexpression of DOK7V1. Moreover, a contrasting effect was seen in adhesion of these cell lines to an artificial basement membrane, with DOK7V1 enhancing the adhesiveness of these lung cancer cell lines. Our further investigations showed a reduced activation of Akt in the lung cancer cell lines following the overexpression of DOK7 variant 1 . However, how DOK7 protein coordinates signalling through Akt pathway and what implication DOK7-regulated Akt pathway has in lung cancer are yet to be investigated in future study.

In conclusion, DOK7 transcripts were reduced in lung cancer and the reduced DOK7 transcript levels were associated with poorer OS and PFS. DOK7 protein isoform 1 was more abundantly expressed in normal lung tissues and was reduced in lung cancers. Overexpression of the DOK7 isoform 1 inhibited proliferation and migration of lung cancer cells in which a reduced activation of Akt was observed, but enhanced the adhesion of lung cancer cells to extracellular matrix. Further investigations are needed to shed light on the interaction between DOK7 and RTKs, including the RTK-mediated signalling that enhanced as a result of DOK7 downregulation, thus leading to disease progression and relapse.

\section{Acknowledgements}

The authors wish to thank Cancer Research Wales, Life Sciences Research Network Wales (Welsh Government's Ser Cymru program) and Albert Huang Foundation. Mr. Gang Chen is a recipient of the China Medical Scholarship from Cardiff University. This study was supported by the National Natural Science Foundation of China (no. 81672726).

\section{References}

1. Siegel RL, Miller KD and Jemal A: Cancer statistics, 2015. CA Cancer J Clin 65: 5-29, 2015.

2. Ferlay J, Soerjomataram I, Dikshit R, Eser S, Mathers C, Rebelo M, Parkin DM, Forman D and Bray F: Cancer incidence and mortality worldwide: Sources, methods and major patterns in GLOBOCAN 2012. Int J Cancer 136: E359-E386, 2015.

3. Yang M, Lewinska M, Fan X, Zhu J and Yuan ZM: PRR14 is a novel activator of the PI3K pathway promoting lung carcinogenesis. Oncogene 35: 5527-5538, 2016.

4. Lemmon MASJ and Schlessinger J: Cell signaling by receptor tyrosine kinases. Cell 141: 1117-1134, 2010.

5. Blume-Jensen P and Hunter T: Oncogenic kinase signalling. Nature 411: 355-365, 2001.

6. Ullrich A and Schlessinger J: Signal transduction by receptors with tyrosine kinase activity. Cell 61: 203-212, 1990.

7. Berger AH, Niki M, Morotti A, Taylor BS, Socci ND, Viale A, Brennan C, Szoke J, Motoi N, Rothman PB, et al: Identification of DOK genes as lung tumor suppressors. Nat Genet 42: 216-223, 2010.

8. Simister PC and Feller SM: Order and disorder in large multi-site docking proteins of the Gab family - implications for signalling complex formation and inhibitor design strategies. Mol Biosyst 8: 33-46, 2012.

9. Jones N and Dumont DJ: Recruitment of Dok-R to the EGF receptor through its PTB domain is required for attenuation of Erk MAP kinase activation. Curr Biol 9: 1057-1060, 1999.

10. Bedirian A, Baldwin C, Abe J, Takano T and Lemay S: Pleckstrin homology and phosphotyrosine-binding domain-dependent membrane association and tyrosine phosphorylation of Dok-4, an inhibitory adapter molecule expressed in epithelial cells. J Biol Chem 279: 19335-19349, 2004.

11. Mashima R, Honda K, Yang Y, Morita Y, Inoue A, Arimura S, Nishina H, Ema H, Nakauchi H, Seed B, et al: Mice lacking Dok-1, Dok-2, and Dok-3 succumb to aggressive histiocytic sarcoma. Lab Invest 90: 1357-1364, 2010.

12. Crowder RJ, Enomoto H, Yang M, Johnson EM Jr and Milbrandt J: Dok-6, a Novel p62 Dok family member, promotes Ret-mediated neurite outgrowth. J Biol Chem 279: 42072-42081, 2004.

13. Grimm J, Sachs M, Britsch S, Di Cesare S, Schwarz-Romond T, Alitalo K and Birchmeier W: Novel p62dok family members, dok-4 and dok-5, are substrates of the c-Ret receptor tyrosine kinase and mediate neuronal differentiation. J Cell Biol 154: 345-354, 2001.

14. Cossins J, Liu WW, Belaya K, Maxwell S, Oldridge M, Lester T, Robb S and Beeson D: The spectrum of mutations that underlie the neuromuscular junction synaptopathy in DOK7 congenital myasthenic syndrome. Hum Mol Genet 21: 3765-3775, 2012.

15. An CH, Kim MS, Yoo NJ and Lee SH: Mutational and expressional analysis of a haploinsufficient tumor suppressor gene DOK2 in gastric and colorectal cancers. APMIS 119: 562-564, 2011.

16. Miyagaki H, Yamasaki M, Takahashi T, Kurokawa Y, Miyata H, Nakajima K, Takiguchi S, Fujiwara Y, Mori M and Doki Y: DOK2 as a marker of poor prognosis of patients with gastric adenocarcinoma after curative resection. Ann Surg Oncol 19: 1560-1567, 2012. 
17. Yamanashi $Y$, Higuch $O$ and Beeson D: Dok-7/MuSK signaling and a congenital myasthenic syndrome. Acta Myol 27: 25-29, 2008.

18. Okada K, Inoue A, Okada M, Murata Y, Kakuta S, Jigami T, Kubo S, Shiraishi H, Eguchi K, Motomura M, et al: The muscle protein Dok-7 is essential for neuromuscular synaptogenesis. Science 312: 1802-1805, 2006.

19. Hamuro J, Higuchi O, Okada K, Ueno M, Iemura S, Natsume T, Spearman H, Beeson D and Yamanashi Y: Mutations causing DOK7 congenital myasthenia ablate functional motifs in Dok-7. J Biol Chem 283: 5518-5524, 2008.

20. Hallock PTXC, Xu CF, Park TJ, Neubert TA, Curran T and Burden SJ: Dok-7 regulates neuromuscular synapse formation by recruiting Crk and Crk-L. Genes Dev 24: 2451-2461, 2010.

21. Bergamin E, Hallock PT, Burden SJ and Hubbard SR: The cytoplasmic adaptor protein Dok7 activates the receptor tyrosine kinase MuSK via dimerization. Mol Cell 39: 100-109, 2010.

22. Inoue A, Setoguchi K, Matsubara Y, Okada K, Sato N, Iwakura Y, Higuchi $\mathrm{O}$ and Yamanashi Y: Dok-7 activates the muscle receptor kinase MuSK and shapes synapse formation. Sci Signal 2: ra7, 2009.

23. Tezuka T, Inoue A, Hoshi T, Weatherbee SD, Burgess RW, Ueta R and Yamanashi Y: The MuSK activator agrin has a separate role essential for postnatal maintenance of neuromuscular synapses. Proc Natl Acad Sci USA 111: 16556-16561, 2014.

24. Ohkawara B, Cabrera-Serrano M, Nakata T, Milone M, Asai N, Ito K, Ito M, Masuda A, Ito Y, Engel AG, et al: LRP4 third $\beta$-propeller domain mutations cause novel congenital myasthenia by compromising agrin-mediated MuSK signaling in a positionspecific manner. Hum Mol Genet 23: 1856-1868, 2014.
25. Heyn H, Carmona FJ, Gomez A, Ferreira HJ, Bell JT, Sayols S, Ward K, Stefansson OA, Moran S, Sandoval J, et al: DNA methylation profiling in breast cancer discordant identical twins identifies DOK7 as novel epigenetic biomarker. Carcinogenesis 34: 102-108, 2013.

26. Lu TP, Tsai MH, Lee JM, Hsu CP, Chen PC, Lin CW, Shih JY, Yang PC, Hsiao CK, Lai LC, et al: Identification of a novel biomarker, SEMA5A, for non-small cell lung carcinoma in nonsmoking women. Cancer Epidemiol Biomarkers Prev 19: 2590-2597, 2010

27. Szász AM, Lánczky A, Nagy Á, Förster S, Hark K, Green JE, Boussioutas A, Busuttil R, Szabó A and Győrffy B: Crossvalidation of survival associated biomarkers in gastric cancer using transcriptomic data of 1,065 patients. Oncotarget 7: 49322-49333, 2016.

28. Chitale D, Gong Y, Taylor BS, Broderick S, Brennan C, Somwar R, Golas B, Wang L, Motoi N, Szoke J, et al: An integrated genomic analysis of lung cancer reveals loss of DUSP4 in EGFR-mutant tumors. Oncogene 28: 2773-2783, 2009.

29. Müller JS, Herczegfalvi A, Vilchez JJ, Colomer J, Bachinski LL, Mihaylova V, Santos M, Schara U, Deschauer M, Shevell M, et al: Phenotypical spectrum of DOK7 mutations in congenital myasthenic syndromes. Brain 130: 1497-1506, 2007. Attribution 4.0 International (CC BY 4.0) License. 